\title{
Intercurrent illness in inborn errors of intermediary metabolism
}

\author{
Marjorie A Dixon, J V Leonard
}

\begin{abstract}
Metabolic decompensation may occur in patients with disorders of intermediary metabolism during intercurrent illness. To prevent complications it is normal practice to change the diet to an 'emergency regimen'. The mainstay of this is a high carbohydrate intake, using soluble glucose polymer, given as frequent drinks by day and during the night. Additional therapy is given for some disorders. Practical details of the treatment are outlined.
\end{abstract}

(Arch Dis Child 1992;67:1387-91)

Diet is the mainstay of treatment for many inborn errors of intermediary metabolism. Patients are stabilised on a diet appropriate for their disorder that will provide them with sufficient nutrients and energy to grow and develop normally. However metabolic stress such as intercurrent infections may precipitate decompensation and cause complications. To prevent these it is common for the diet to be changed to an 'emergency regimen'. The purpose of this paper is to outline the principles and practice of these regimens.

\section{Principles}

During illness and fasting various metabolic adaptations occur. ${ }^{2}$

\section{(A) PROTEIN CATABOLISM}

There is constant turnover of tissue protein and overall, the rate of protein synthesis exceeds that of breakdown so there is net gain and hence growth. ${ }^{3}$ However, during illness the rate of protein breakdown normally exceeds that of synthesis with net production of amino acids and an increase in their irreversible catabolism. The nitrogen moiety of amino acids is converted to ammonia and then to urea. The carbon skeleton of amino acids is progressively catabolised to form fuels for energy production including acetyl CoA, pyruvate, and intermediates of the Krebs cycle. Sick Children and The Institute of Child Health, London Mariorie A Dixon Marjorie A D Correspondence to: Professor J V Leonard Professor J V Leonard, Medical Unit, Institute of Child Health Guilford Street, during fasting are relatively limited in children. To maintain a supply of substrate for energy production and protect glucose supply to the brain it is necessary to mobilise alternative fuels, including free fatty acids, ketones, and gluconeogenic precursors.

On fasting, as glucose concentrations slowly fall, there is a decrease in the insulin:glucagon ratio with mobilisation of fatty acids from adipose tissue stores. Free fatty acids can be utilised by many tissues, such as the heart and skeletal muscle, but they cannot enter the brain. In the liver, free fatty acids are partially oxidised to ketones which are water soluble and can enter the central nervous system. Alanine and other amino acids from muscle catabolism and glycerol from lipolysis are substrates for gluconeogenesis.

The purpose of the emergency regimen is to prevent the changes that occur with fasting. The aim is to reduce protein catabolism and hence the accumulation of potentially toxic metabolites. By giving an adequate supply of glucose the mobilisation of alternative fuels is also reduced.

\section{Practical aspects of emergency regimens}

The core of the emergency regimen is essentially similar for all disorders. A solution of glucose polymer is given as the major source of energy because it is simple, palatable, and usually well tolerated. Fat emulsions can provide additional energy, but these are less well tolerated; fat delays gastric emptying and is more likely to cause vomiting so we do not use them routinely. It is also contraindicated in some disorders, such as inborn errors of fatty acid oxidation. In most instances we would start by giving feeds orally. There are important advantages to oral feeding. It can be started at home; more glucose can be given than by peripheral intravenous infusions and medicines can be given. This can be important as intravenous preparations of the medicine are often not readily available. If oral feeding is not possible, nasogastric feeding, either bolus or continuous, should be tried before intravenous therapy for the same reasons.

The concentrations and volumes of solution given will depend on the age of the child. Relatively higher volumes and lower concentrations are used in infants compared with older children (table). Insufficient fluid combined with high concentrations of glucose polymer that can cause diarrhoea may exacerbate the effects of illness. If the child is likely to become dehydrated it is advisable to give an oral rehydration solution supplemented with glucose 
Emergency regimen

\begin{tabular}{|c|c|c|c|c|}
\hline \multirow[t]{2}{*}{$\begin{array}{l}\text { Age } \\
\text { (years) }\end{array}$} & \multicolumn{2}{|c|}{$\begin{array}{l}\text { Glucose polymer } \\
\text { concentration }\end{array}$} & \multirow[t]{2}{*}{ Daily volume } & \multirow[t]{2}{*}{ Frequency } \\
\hline & $\%$ & kcall/100 ml* & & \\
\hline $\begin{array}{l}0-1 \\
1-2 \\
2-6 \\
6-10 \\
>10\end{array}$ & $\begin{array}{l}10 \\
15 \\
20 \\
20 \\
25\end{array}$ & $\begin{array}{r}40 \\
60 \\
80 \\
80 \\
100\end{array}$ & $\begin{array}{l}150-200 \mathrm{ml} / \mathrm{kg} \\
95 \mathrm{ml} / \mathrm{kg} \\
1200-1500 \mathrm{ml} \\
1500-2000 \mathrm{ml} \\
2000 \mathrm{ml}\end{array}$ & $\begin{array}{l}\text { Initially every } \\
\text { two hours } \\
\text { night and day }\end{array}$ \\
\hline
\end{tabular}

${ }^{*} 1 \mathrm{kcal}=4 \cdot 18 \mathrm{~kJ}$

polymer to the required concentration. (Practice note: oral rehydration solutions do not contain sufficient glucose on their own.) The solution is given orally as small frequent drinks or enterally via a nasogastric tube, normally at intervals of two hours night and day. This is usually well tolerated. It is particularly important not to allow the child to go for long periods ( $>4$ hours) between drinks during the night.

Parents are taught to make solutions using an appropriate sized scoop which is quick and easy. These solutions can also be frozen and thawed when needed at home or in hospital. All proprietary liquid glucose polymer solutions have a concentration of $50 \%$ carbohydrate or more, for example, Fortical (Cow and Gate) and Liquid Maxijul (Scientific Hospital Supplies), and are not suitable for an emergency regimen unless diluted to an appropriate concentration. In our experience these are less palatable and more likely to induce vomiting or diarrhoea if given undiluted.

In some children the first symptom of any illness is refusal to eat or drink. If so, we often teach the parents how to use a nasogastric tube at home, thereby reducing the need for a hospital admission.

If the child has occasional vomits, it may still be possible to feed orally by giving the drinks as frequent sips (for example $10 \mathrm{ml}$ every 10 minutes) or as a continuous nasogastric feed either at home or in hospital. However, if the child is vomiting frequently or is obviously unwell then intravenous therapy is essential. Concentrated glucose solutions should be used: $10 \%$ dextrose by peripheral drip or more concentrated through a central line. Hyperglycaemia may develop so blood glucose concentrations should be monitored regularly.

\section{Instructions for parents}

Many parents have difficulty in knowing both when to start the emergency regimen and exactly what to do. To overcome this it is our practice to teach a three stage approach.

(1) If the parents are uncertain whether their child is unwell (or just tired) because they appear lethargic, irritable, or off colour then an emergency regimen drink should be given. Next, a conscious decision is made to reassess the child within one to four hours, depending on the age and disorder. In some children it is possible to monitor the disorder by using simple tests at home. These are discussed later under the individual disorders.

(2A) If on review the child has improved, the normal diet is resumed. (B) If on reassessment there is no improvement the full emergency regimen of drinks every two hours should be started. There is some flexibility in the frequency of the drinks particularly in the older children and during recovery. During illness most children will automatically stop eating the normal diet, and once the child starts to improve the usual diet is gradually reintroduced. (3) If the child is refusing to take the drinks, is vomiting frequently, or becoming encephalopathic then they should be admitted to hospital for assessment. The parents need to be aware of and recognise clinical signs of deterioration. Of particular importance is the ability to recognise encephalopathy with the child becoming less responsive, often with a glazed look.

Parents may face difficulties if the child does not take all the recommended volumes of feed. The quantities necessary vary both with the underlying inborn error and the intercurrent illness. It is particularly important that patients with maple syrup urine disease and methylmalonic acidaemia should have close to the recommended volumes (see below). By contrast those with glycogen storage disease may be controlled satisfactorily with smaller volumes. Patients with gastroenteritis will in general need more fluid than those with upper respiratory tract infections.

It should be emphasised that the basic emergency regimen must not be continued for long periods of time because it does not provide adequate nutrition and will cause nutritional deficiencies. Early clinical signs of such deficiencies include skin rashes (particularly at the site of adhesive tape for nasogastric tubes). Most patients can be gradually returned to their usual diet within a few days.

When the diet is being reintroduced additional high carbohydrate drinks are given until the normal diet is re-established. In infants, additional glucose polymer is added to the formula feed. For patients on low protein diets the protein intake is usually increased daily, giving one quarter, one half, three quarters of the usual intake, resuming the normal protein allowance by day four.

Whenever the child has been ill it is usual for us to discuss the emergency regimen carefully, checking that the parents knew what to do and that the emergency regimen meets the needs of their child. It is important to recognise that the course of these disorders is often unpredictable. The children may have a serious infection without any problems, but then develop severe decompensation after apparently minimal stress; therefore the need for careful management of all intercurrent illness.

\section{Specific treatment}

This basic emergency regimen is suitable for disorders of carbohydrate metabolism including glycogen storage diseases, fructose-1, 6-diphosphatase deficiency, and 'ketotic hypoglycaemia'. However, in other metabolic disorders the emergency regimen is combined with specific treatment.

MAPLE SYRUP URINE DISEASE (MSUD)

In MSUD the branched chain ketoacid dehydrogenase is defective. This enzyme is the 
second step on the catabolic pathway of the three branch chain amino acids (BCAA): leucine, isoleucine, and valine. This pathway is the major route for disposal of the BCAA. As it is blocked in MSUD $^{4}$ the BCAA and respective ketoacids accumulate and are responsible for the encephalopathy. Losses of BCAA and ketoacids in the urine and through other pathways are low ${ }^{4}$ so the only way to reduce plasma concentrations is for the BCAA to be incorporated into protein. The objective of treatment during illness is to increase the rate of protein synthesis by giving a high energy intake as glucose with or without fat. Although the rate of protein synthesis may increase initially the concentration of the essential amino acids other than the BCAA will soon become rate limiting. To prevent this supplements of the BCAA free

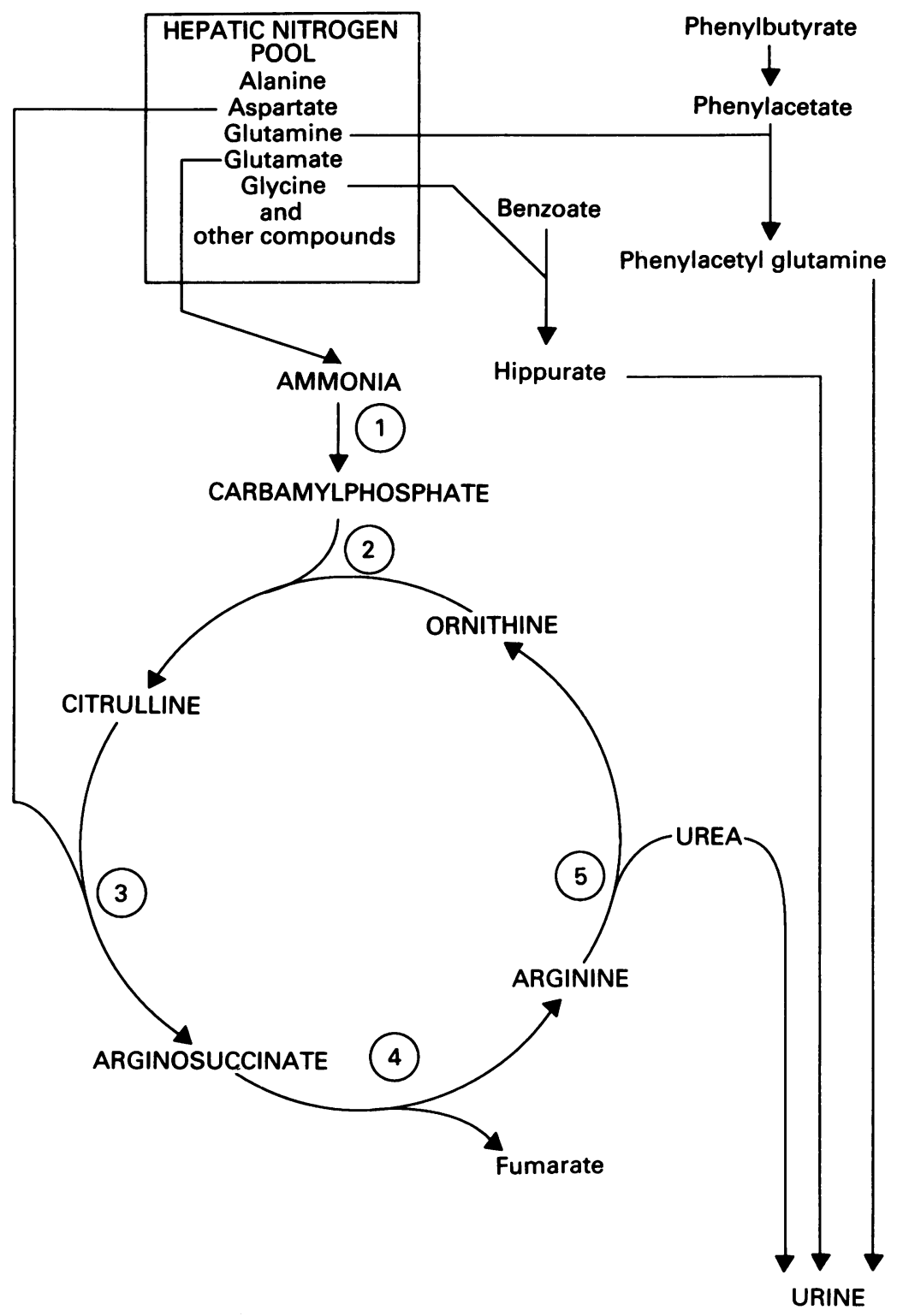

Hepatic nitrogen metabolism. Urea, the major product of nitrogen catabolism, is synthesised within the urea cycle from aspartate and ammonia. Defects of each step in the cycle have been identified. (1) Carbamylphosphate synthetase deficiency. (2) Ornithine carbamyltransferase deficiency. (3) Arginosuccinate synthetase deficiency (citrullinaemia). (4) Arginosuccinate lyase deficiency (arginosuccinic aciduria). (5) Arginase deficiency. In these disorders sodium benzoate and sodium phenylacetate (or phenylbutyrate) are used to reduce the flux in the urea cycle. Benzoate is conjugated with the glycine to form hippurate and phenylacetate conjugated with glutamine to form phenylacetyl glutamine. Both of these compounds are rapidly excreted in the urine reducing the accumulation of ammonia and amino acids. amino acids should be given. ${ }^{5}$ Our aim is to provide the full energy requirement and the child's usual quantity of BCAA free supplement. This is not always possible orally, particularly if the child is vomiting. A useful alternative is continuous nasogastric feeding of Maxamaid MSUD (Scientific Hospital Supplies, see appendix) supplemented with extra glucose polymer. We have successfully used this to treat patients with MSUD who have been unable to tolerate bolus feeds. Parenteral nutrition, using a BCAA free amino acid solution, is an alternative if enteral feeding fails but this is available in few centres. Regular daily quantitative measurements of the BCAA are essential to monitor progress and to determine when protein can be reintroduced.

Once the plasma leucine concentration falls below $800 \mu \mathrm{mol} / \mathrm{l}$ some leucine usually as natural protein can be reintroduced, and is increased to the usual intake according to the plasma concentrations. Our aim is to keep plasma leucine between $200-700 \mu \mathrm{mol} / 1$. During the recovery phase concentrations of the isoleucine and valine may fall to low concentrations and become rate limiting for protein synthesis. Supplementation of these amino acids then becomes necessary, in doses of 50-300 mg/day. To do this a solution of the amino acids is made (providing $100 \mathrm{mg}$ of amino acid in $10 \mathrm{ml}$ ) and the required amount added to the feed. We do not routinely give valine and isoleucine supplements ${ }^{5}$ as not all patients need them and this practice could cause imbalance in amino acid concentrations. Hypokalaemia may develop during the recovery phase.

\section{PHENYLKETONURIA (PKU)}

Many centres do not give patients with PKU an emergency regimen or monitor plasma phenylalanine concentrations during illness. However with increasing emphasis on improving metabolic control, an emergency regimen is likely to be necessary. It should be similar to that used in MSUD.

\section{UREA CYCLE DISORDERS}

The urea cycle converts waste nitrogen into urea, via a series of enzymes (figure). Inborn errors have been identified for each step and may cause accumulation of ammonia and glutamine which are neurotoxic and may cause a severe encephalopathy.

Patients are treated with a low protein diet and medicines which promote nitrogen excretion via alternative pathways. Sodium benzoate is most widely used. It is conjugated with glycine to form hippurate, 1 mole of nitrogen being excreted for each mole of sodium benzoate given. Phenylbutyric acid can also be given either as the free acid or the sodium salt but is less palatable. Phenylbutyrate is metabolised in vivo to phenylacetate, and is more effective than benzoate because it is conjugated with glutamine to form phenylacetylglutamine, 2 moles of nitrogen being excreted for each mole of phenylbutyrate given.

During illness protein breakdown may cause 
rapid accumulation of ammonia and glutamine. In addition to the basic emergency regimen the usual medication should continue to be given. Both sodium benzoate and phenylbutyrate are given in a dose of $250 \mathrm{mg} / \mathrm{kg} /$ day but can be temporarily increased to $500 \mathrm{mg} / \mathrm{kg} /$ day during illness.

Another consequence of these disorders is that arginine becomes an essential amino acid (except in arginase deficiency). During illness the normal arginine supplements, $100 \mathrm{mg} / \mathrm{kg} /$ day, should be given. In citrullinaemia and argininosuccinic aciduria, arginine should be given in larger doses of up to $700 \mathrm{mg} / \mathrm{kg} /$ day to replenish ornithine that is not reformed as a result of the metabolic block. All the medication should be given in frequent small doses. If the child cannot tolerate oral fluids or medicines sodium benzoate, sodium phenylacetate, and arginine should be given intravenously.

Patients should be monitored with regular measurement of plasma ammonia and quantitative plasma amino acids. Protein can be reintroduced once the ammonia is less than $80 \mu \mathrm{mol} / \mathrm{l}$.

Occasionally it can be difficult to reintroduce protein without inducing hyperammonaemia and in these cases an essential amino acid supplement (for example Dialamine, Scientific Hospital Supplies, see appendix), can be given. This will promote both protein synthesis and the reutilisation of nitrogen to form nonessential amino acids thereby reducing ammonia accumulation.

\section{TYROSINAEMIA TYPE I (FUMARYLACETOACETASE DEFICIENCY)}

Tyrosinaemia type $I$ is caused by a deficiency of fumarylacetoacetase, the last enzyme of the catabolic pathway of tyrosine, with resultant accumulation of succinylacetone and related metabolites. Patients are usually treated with a diet restricted in tyrosine and phenylalanine. Although it is not common to use an emergency regimen in this disorder, decompensation of liver function and deterioration of neurological function can follow intercurrent infection. It is probably beneficial that an emergency regimen is given to reduce these problems. The patients may also need appropriate treatment for liver failure.

\section{ORGANIC ACIDAEMIAS}

(a) Propionic and methylmalonic acidaemia

Propionic and methylmalonic acidaemia are caused by inherited enzyme defects in the pathways of propionate catabolism. Propionate is formed from several sources including the amino acids isoleucine, valine, methionine, and threonine, by anaerobic bacterial fermentation in the gut and catabolism of odd chain fatty acids. Although amino acids are widely regarded as the major source of propionate, only about $50 \%$ of propionate is derived from breakdown of amino acids. ${ }^{6}$ About $20 \%$ is derived from the gut $^{6}$ and the rest $(30 \%)$ probably from the catabolism of odd chain fatty acids. ${ }^{7}$ The toxic metabolites and the mechanisms responsible for all the clinical problems are not well understood but the aim of treatment is to reduce the accumulation of propionate by diminishing production and increasing disposal.

In addition to the standard emergency regimen to reduce protein breakdown and amino acid catabolism, propionate production from the gut is reduced by giving metronidazole (or an alternative antibiotic). The removal of propionyl groups is enhanced by giving L-carnitine. This forms propionyl carnitine which is excreted in the urine. Carnitine is given either orally or intravenously in a dose of $100 \mathrm{mg} / \mathrm{kg} / \mathrm{day}$. Higher doses have been used although their value and complications have not been assessed critically. In methylmalonic acidaemia there is usually massive excretion of methylmalonate in the urine with obligatory simultaneous losses of sodium and potassium. These patients also have a concentrating and acidification defect in the kidney ${ }^{8}$ so it is essential to give sufficient fluid with supplements of sodium bicarbonate (2-3 $\mathrm{mmol} / \mathrm{kg}$ ) to replace sodium loss and reduce the acidosis. Patients may also need potassium supplements particularly during the recovery phase.

It may be helpful to monitor the patient's condition by measuring urine ketones but in our experience the parents' clinical judgment is as useful for most children.

It is important to reintroduce protein early (within two to three days) to prevent protein deficiency and additional problems such as rashes and vomiting.

\section{(b) Isovaleric acidaemia}

Isovaleric acidaemia is due to a deficiency of isovaleryl-CoA dehydrogenase, the third step in the pathway of leucine degradation. Isovaleric acid accumulates and its excretion in the urine can be increased by giving L-carnitine $(100 \mathrm{mg} /$ $\mathrm{kg} /$ day $)$ and glycine $(250 \mathrm{mg} / \mathrm{kg} /$ day $)$. These are conjugated to form isovalerylcarnitine and isovalerylglycine respectively, both being rapidly excreted in the urine. This treatment can be given orally or intravenously.

(c) Other organic acidaemias (for example, 3-methylcrotonylglycinuria, glutaric aciduria type I)

In addition to the standard emergency regimen, $\mathrm{L}$-carnitine is widely used to increase the removal of acyl groups, although controlled studies of its efficacy are lacking.

\section{DISORDERS OF FATTY ACID OXIDATION}

Inborn errors at several steps in the pathway of fatty acid oxidation are now well described, the most common is medium chain acyl CoA dehydrogenase deficiency. To prevent illness in these patients the standard emergency regimen is used. It is important to stress early use of the emergency regimen to inhibit mobilisation of fatty acids, particularly in long chain disorders because decompensation may be rapid. Additional treatment with $\mathrm{L}$-carnitine is widely used but is somewhat controversial; there is little detailed critical work. Carnitine is essential in 
primary systemic carnitine deficiency and may be helpful in reversing decompensation in disorders of medium chain fatty acids. In theory carnitine in long chain fatty acid oxidation disorders might be harmful, by promoting the uptake of long chain fatty acids into mitochondria. Its use requires more evaluation.

Blood glucose is often used to monitor patients with these disorders, but this may give a false sense of security as patients may develop marked encephalopathy before blood glucose concentrations fall: treatment must be started before this.

\section{Conclusions}

The use of a well planned emergency regimen will not only help prevent episodes of metabolic decompensation, but will reduce hospital admissions and improve the parents' self confidence. The policy of encouraging the family to bring the unwell child to hospital every time is intended to be supportive and may initially be necessary, but it may add to the burden of care. The correct balance needs to be found between overzealous intervention and the risk of complications. Furthermore, the needs of each child and their family must be judged carefully and the instructions adjusted to their own individual requirements.

We would like to thank Dr Peter Clayton and Dr Margaret Lawson for their constructive criticism of the manuscript.

Note: References about the inborn errors have, in general, only been given where the statement is not substantiated in Scriver et al. ${ }^{1}$

1 Scriver CR, Beaudet AL, Sly WS, Valle D, eds. The metabolic basis of inherited disease. 6th Ed. New York: McGraw-Hill, 1989.

2 Newsholme EA, Leech AR. Biochemistry for the medical sciences. Chichester: John Wiley, 1983.

3 Rennie MJ, Edwards RHT, Halliday D, Matthews DE, Wolman SL, Milward DJ. Muscle protein synthesis measured by stable isotope techniques: the effects of feeding and fasting. Clin Sci 1982;63:519-23.

4 Thompson GN, Bresson JL, Walter JH, et al. Protein and leucine metabolism in maple syrup urine disease. $A m \mathcal{F}$ Physiol 1990;254:654 60.

5 Thompson GN, Francis DEM, Halliday D. Acute illness in maple syrup urine disease: dynamics of protein metabolism and implications for mangement. $\mathcal{f}$ Pediatr 1991;119: and im 41 .

6 Thompson GN, Walter JH, Bresson JL, et al. Sources of propionate production in inborn errors of propionate metabolism. Metabolism 1990;39:1133-7.

7 Sbai D, Prudhom C, Thompson GN, Mariotti A, Saudubray JM, Bresson JL. Possible contributions of odd chain fatty acid oxidation to propionate production in methylmalonic and propionic acidaemia. Pediatr Res 1992;31:188A

8 D'Angio C, Leonard JV, Dillon MJ. Renal tubular function in methylmalonic acidaemia. Eur 7 Pediatr 1991;150: $259-63$.

Appendix Manufactured products used in maple syrup urine disease (MSUD) and urea cycle disorders (UCD)

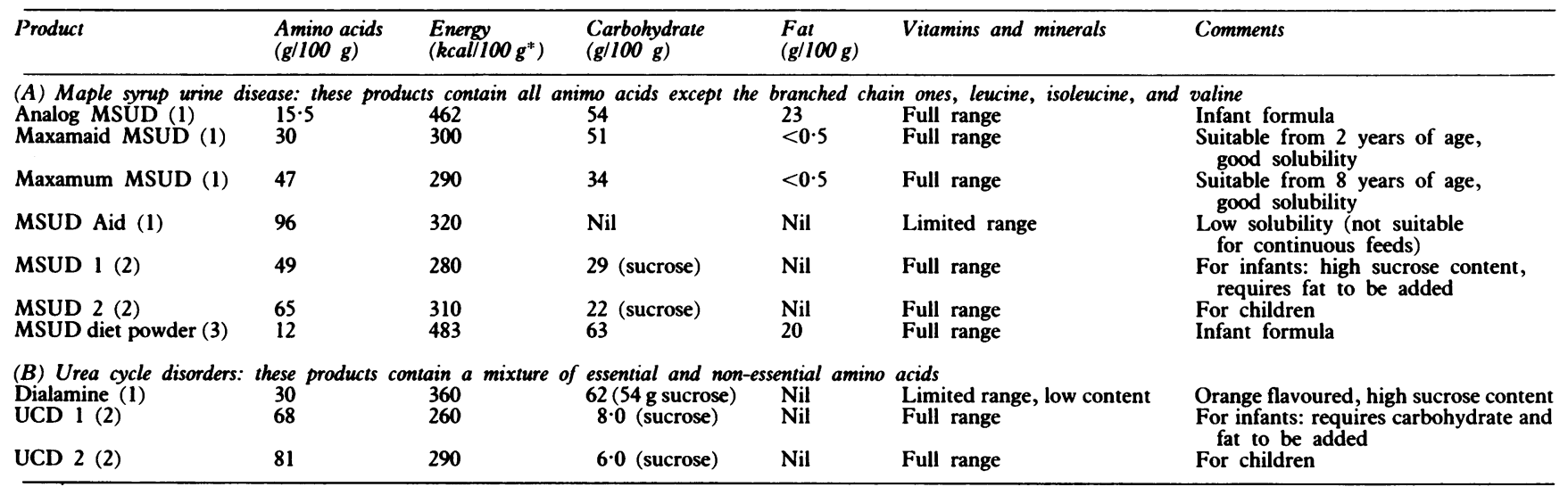

Manufacturer: (1) Scientific Hospital Supplies (SHS), Liverpool; (2) Milupa, Hillingdon, Middlesex; (3) Mead Johnson Nutritionals, Hounslow, Middlesex. In the UK:

Only the SHS products have been approved by the Advisory Committee Borderline Substances and can be prescribed on an FP10.
${ }^{1} 1 \mathrm{kcal}=4 \cdot 18 \mathrm{~kJ}$. 